\title{
First prototypes of vortex retarders obtained by polarization holography
}

P. Piron, P. Blain, M. Décultot, D. Mawet, S. Habraken

P. Piron, P. Blain, M. Décultot, D. Mawet, S. Habraken, "First prototypes of vortex retarders obtained by polarization holography," Proc. SPIE 9099, Polarization: Measurement, Analysis, and Remote Sensing XI, 909911 (21 May 2014); doi: 10.1117/12.2052830

SPIE Event: SPIE Sensing Technology + Applications, 2014, Baltimore, Maryland, United States 


\title{
First prototypes of vortex retarders obtained by polarization holography
}

\author{
Piron P. ${ }^{a *}$ Blain P. ${ }^{a}$, Décultot M. ${ }^{a}$, Mawet D. ${ }^{b}$, Habraken S. ${ }^{a}$ \\ ${ }^{a}$ Hololab, University of Liège, 17 Allée du 6 Août, 4000 Liège, Belgium; \\ ${ }^{b}$ European Southern Observatory, Alonso de Córdova 3107, Vitacura 7630355, Santiago, Chile
}

\begin{abstract}
This paper will present the first prototypes of vortex retarders made of photo-orientable liquid crystals polymers recorded without mechanical action using only polarization holography.

Vortex retarders are birefringent plates characterized by a uniform phase retard and a rotation of their fast axis along their center.

Liquid crystals are anisotropic molecules possessing birefringent properties. They are locally orientable and their orientation defines the fast axis orientation of the retarder. Their alignment depends on the local orientation of the recording electric field. The superimposition of several polarized beams will be used to shape the electric field to achieve the recording of vortex retarders.

The mathematical aspects of the superimposition process, as well as several numerical simulations are exposed. Finally, the first prototypes are presented, characterized and compared to the numerical simulations.
\end{abstract}

Keywords: Polarization, Holography, Liquid Crystal Polymers, Vectorial vortex

\section{INTRODUCTION}

Vortex retarders are birefringent plate characterized by a rotation of their fast axis (Figure 1 (a)). The goal of the plate is to create a vortex beam i.e. a beam with phase dislocation of the form $e^{\imath l \theta}$ (Figure 1 (b)) where $\theta$ is the polar angle inside the beam and $l$ the topological charge. ${ }^{1,2}$

These beams could be used for coronagraphy, ${ }^{2-4}$ creation of highly focused beams, ${ }^{5,6}$ optical tweezers, ${ }^{6,7} \ldots$ In a previous paper, ${ }^{8}$ we numerically demonstrated that the vortex retarders in liquid crystal polymers (LCP) could be recorded using the superimposition of differently polarized beams.

In this paper, we will present the first prototypes of vortex retarders composed of liquid crystals polymers recorded using polarization holography.

Section 2 focuses on the recording process of the retarders. It will present the generic recording of the retarders and the mathematical model of polarization holography and its application to the recording of vortex retarders. Section 3 describes the recording setup. The elements composing the setup and their effects on the beam and its polarization state will be detailed. It also exposes the measurements of the recording electric field. The essential features of the electric field will be showed as well as the simulations of the expected retarder characteristics. Section 4 presents the first two prototypes and will compare them to the expected retarder. To conclude, a summary of the research and the future developments will be presented.

*FRIA PhD Scholar

Further author information: (Send correspondence to Piron P.)

Piron P.: E-mail: pierre.piron@ulg.ac.be

Polarization: Measurement, Analysis, and Remote Sensing XI, edited by David B. Chenault, Dennis H. Goldstein, Proc. of SPIE Vol. 9099, 909911 · @ 2014 SPIE · CCC code: 0277-786X/14/\$18 · doi: 10.1117/12.2052830 


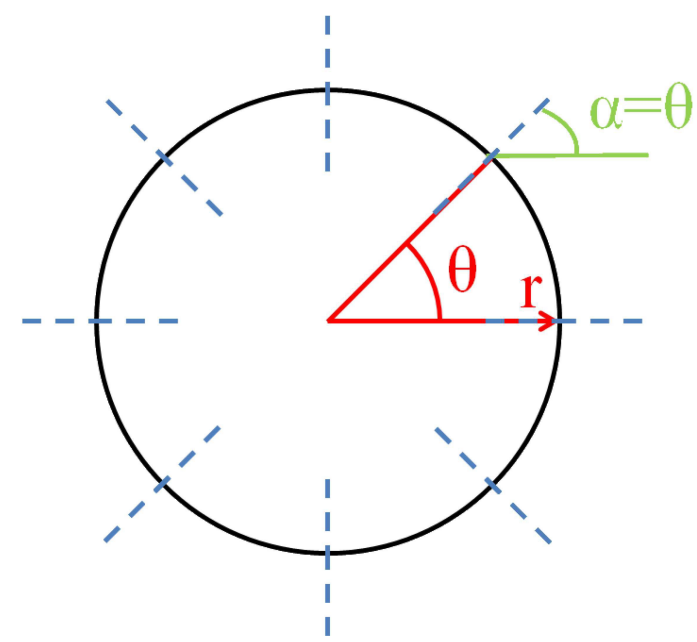

(a)

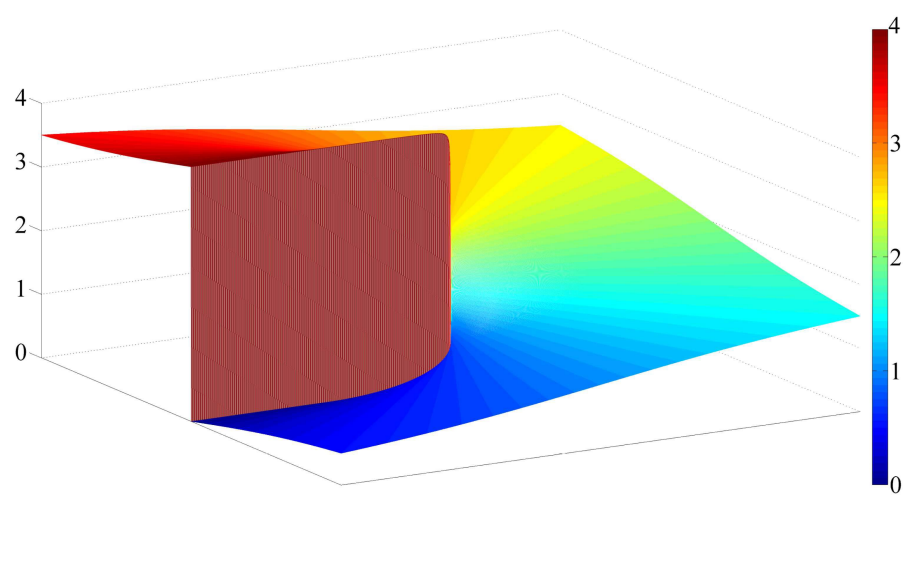

(b)

Figure 1. (a) Representation of a charge 2 vortex, the fast axis is pictured by dashed blue lines and its orientation is a multiple of the polar angle $\theta$. (b) Representation of the phase induced by the retarder as a multiple of $\pi$.

\section{RECORDING OF VORTEX RETARDERS IN LCP USING POLARIZATION HOLOGRAPHY}

The recording of our retarders in LCPs consists in two steps.

- The first step consists in the exposure of an alignment layer (ROLIC@: ROP-103). The layer is spin-coated on a glass substrate. It contains photo-sensitive polymers that align themselves according to the direction of the incident polarization. The layer is exposed to UV linearly polarized beam. The orientation of the incident polarization will define the orientation of the fast axis ofthe future retarder.

- The second step uses a liquid crystal pre-polymer (ROLIC@: ROF-5102). The layer is spin-coated on the first one. It contains the liquid crystals which align themselves according to the orientation of the polymers below. The thickness of the second layer will define the phase retard $\Delta \phi$ according to $\Delta \phi=\frac{2 \pi \Delta n h}{\lambda}$. Where $\Delta n$ is the birefringence, $h$ the thickness of the layer and $\lambda$ is the incident wavelength. Presently, the spin-coating parameters of the second layer are chosen to reach a half-wave plate near $550 \mathrm{~nm}$.

To record vortex retarders, an electric field with a rotation of the local polarization must be achieved. The superimposition of differently polarized beams will be used to shape the electric field.

Kilosanidze et al. ${ }^{9}$ developed a mathematical model of the local polarization resulting from the superimposition of two generic coherent beams based on the Jones formalism. ${ }^{10}$

Two generic coherent beams $A$ and $B$ are represented by the following equation.

$$
A=\left(\begin{array}{c}
A_{x} \\
A_{y} \exp \left(i \phi_{A}\right)
\end{array}\right) \exp (i \omega t) B=\left(\begin{array}{c}
B_{x} \\
B_{y} \exp \left(i \phi_{B}\right)
\end{array}\right) \exp (i \delta) \exp (i \omega t)
$$

Where $A_{x}$ and $B_{x}$ are the amplitude modules of the horizontal component of $A$ and $B, A_{y}$ and $B_{y}$ the amplitude modules of the vertical components, $\phi_{A}$ and $\phi_{B}$ the phase differences between the horizontal and vertical components. $\omega$ is the pulsation of the beams and $\delta$ is the phase difference between the two beams. 
At the overlap plane, the resulting electric field: $\Sigma$ can be written as equation 2 .

$$
\Sigma=\left(\begin{array}{c}
A_{x}+B_{x} \exp (i \delta) \\
A_{y} \exp \left(i \phi_{A}\right)+B_{y} \exp \left(i \phi_{B}+i \delta\right)
\end{array}\right) \exp (i \omega t)
$$

The local polarization ellipse is described by the real part of the electric field and is written as

$$
\Re(\Sigma)=\mathbf{p} \cos (\omega t)+\mathbf{q} \sin (\omega t)
$$

where $\mathbf{p}=\left(\begin{array}{c}A_{x}+B_{x} \cos (\delta) \\ A_{y} \cos \left(\phi_{A}\right)+B_{y} \cos \left(\phi_{B}+\delta\right)\end{array}\right)$ and $\mathbf{q}=-\left(\begin{array}{c}B_{x} \sin (\delta) \\ A_{y} \sin \left(\phi_{A}\right)+B_{y} \sin \left(\phi_{B}+\delta\right)\end{array}\right)$.

To describe the local polarization ellipse, three parameters must be provided: $I_{1}$ the intensity along the largest axis, $I_{2}$ the intensity along the smallest axis and $\alpha$ the angle between the horizontal and the largest axis of the ellipse (see Figure 2).

These parameters are given by

$$
\begin{gathered}
I_{1,2}=\frac{1}{2}\left[\left(p_{x}^{2}+p_{y}^{2}\right)+\left(q_{x}^{2}+q_{y}^{2}\right)\right] \pm \frac{1}{2} \sqrt{\left[\left(p_{x}^{2}-p_{y}^{2}\right)+\left(q_{x}^{2}-q_{y}^{2}\right)\right]^{2}+4\left(p_{x} p_{y}+q_{x} q_{y}\right)^{2}}, \\
\sin (2 \alpha)=\frac{2\left(p_{x} p_{y}+q_{x} q_{y}\right)}{\sqrt{\left[\left(p_{x}^{2}-p_{y}^{2}\right)+\left(q_{x}^{2}-q_{y}^{2}\right)\right]^{2}+4\left(p_{x} p_{y}+q_{x} q_{y}\right)^{2}}}, \\
\cos (2 \alpha)=\frac{\left(p_{x}^{2}-p_{y}^{2}\right)+\left(q_{x}^{2}-q_{y}^{2}\right)}{\sqrt{\left[\left(p_{x}^{2}-p_{y}^{2}\right)+\left(q_{x}^{2}-q_{y}^{2}\right)\right]^{2}+4\left(p_{x} p_{y}+q_{x} q_{y}\right)^{2}}} .
\end{gathered}
$$

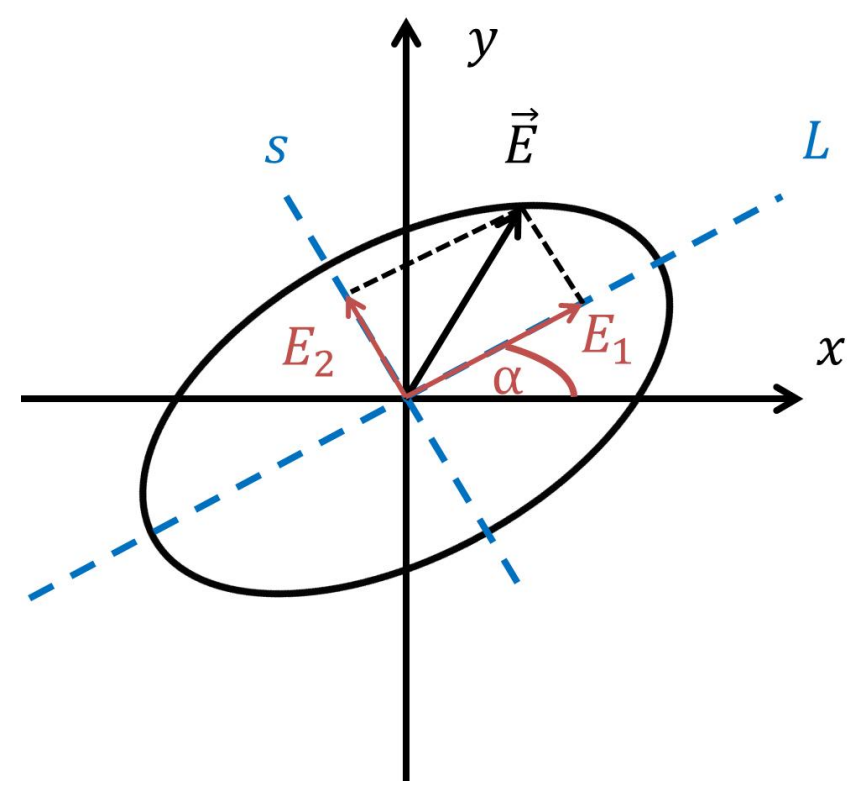

Figure 2. Representation of the local polarization ellipse, the largest (L) and the smallest (s) axis are pictured by dashed blue lines.

Let us notice that $I_{2}=0$ and $I_{1} \neq 0$ always represents a linear polarization and $I_{2}=I_{1} \neq 0$ represents a circular polarization. 
To illustrate the model, two simple cases can be studied:

- the classical case of two linearly polarized beams with the same polarization,

- two circularly polarized beams of opposite handedness.

a: 2 linearly polarized beams with the same polarization: vertical polarization

$$
\begin{aligned}
& A_{x}=B_{x}=0 \\
& A_{y}=B_{y}=1 \\
& \phi_{A}=\phi_{B}=0
\end{aligned} \quad \Rightarrow \quad \begin{aligned}
& I_{1}=2 \times(1+\cos (\delta)) \\
& I_{2}=0 \\
& \alpha=90^{\circ}
\end{aligned}
$$

b: 2 circularly polarized beams of opposite handedness

$$
\begin{aligned}
& A_{x}=B_{x}=\frac{\sqrt{2}}{2} \quad I_{1}=1+|\cos (\delta)| \\
& A_{y}=B_{y}=\frac{\sqrt{2}}{2} \quad \Rightarrow \quad I_{2}=1-|\cos (\delta)| \\
& \phi_{A}=-\frac{\pi}{2}: \phi_{B} a=\frac{\pi}{2} \quad \theta=\frac{\delta}{2}
\end{aligned}
$$

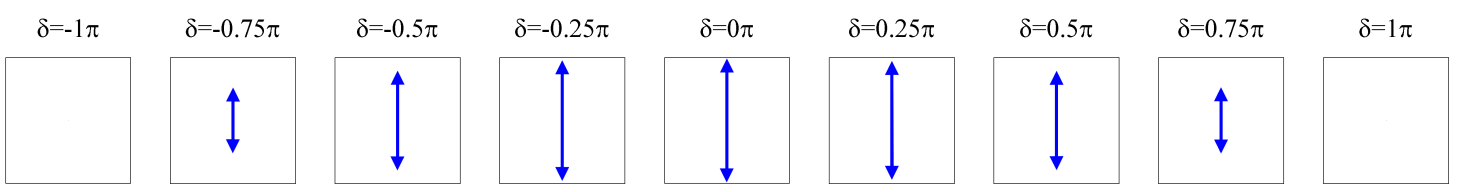

Figure 3. Representation of the local polarization ellipse for case a.
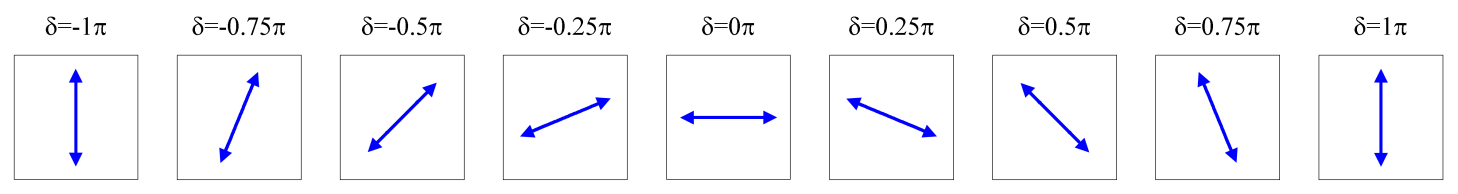

Figure 4. Representation of the local polarization ellipse for case $b$.

As first tests of polarization holography, we recorded the superimposition of two orthogonal circularly polarized beams to achieve a polarization analyzer ${ }^{11}$ and circular polarization gratings for shearography. ${ }^{12}$ 
To record vortex retarders, an electric field with a rotation of the local polarization must be achieved. The superimposition of differently polarized beams will be used to shape the electric field. The geometry of the superimposition is pictured in Figure 5.

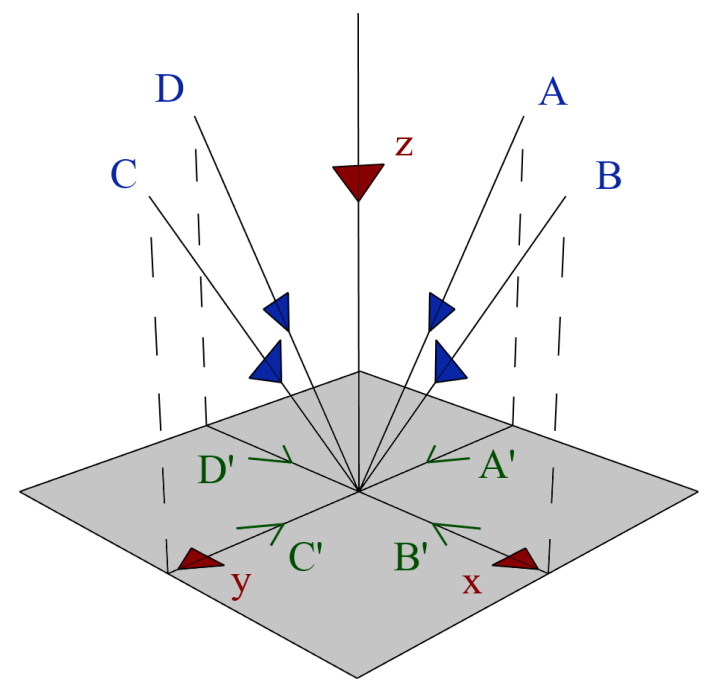

Figure 5. Representation of the superimposition with four beams, possessing the same incident angle but various incident planes.

Using the previous formalism, the four beams are described by:

$$
\begin{aligned}
& A=\left(\begin{array}{c}
A_{x} \\
A_{y} \exp \left(i \phi_{A}\right)
\end{array}\right) \exp \left(i \delta_{A}\right), \quad B=\left(\begin{array}{c}
B_{x} \\
B_{y} \exp \left(i \phi_{B}\right)
\end{array}\right) \exp \left(i \delta_{B}\right) \\
& C=\left(\begin{array}{c}
C_{x} \\
C_{y} \exp \left(i \phi_{C}\right)
\end{array}\right) \exp \left(i \delta_{C}\right), \quad D=\left(\begin{array}{c}
D_{x} \\
D_{y} \exp \left(i \phi_{D}\right)
\end{array}\right) \exp \left(i \delta_{D}\right) .
\end{aligned}
$$

An important modification is that the $\delta_{i}$ of each beam are computed as the phase difference between the $i$ plane-wave beam and a virtual beam with a normal incidence. Therefore

$$
\begin{array}{ll}
\delta_{A}=\frac{2 \pi}{\lambda} \sin \left(\theta_{i}\right) \times(y), & \delta_{B}=\frac{2 \pi}{\lambda} \sin \left(\theta_{i}\right) \times(-x), \\
\delta_{C}=\frac{2 \pi}{\lambda} \sin \left(\theta_{i}\right) \times(-y), & \delta_{D}=\frac{2 \pi}{\lambda} \sin \left(\theta_{i}\right) \times(x),
\end{array}
$$

with $\theta_{i}$ is the incident angle.

The two vectors describing the polarization ellipse become:

$$
\begin{gathered}
\mathbf{p}=\left(\begin{array}{c}
A_{x} \cos \left(\delta_{A}\right)+B_{x} \cos \left(\delta_{B}\right)+C_{x} \cos \left(\delta_{C}\right)+D_{x} \cos \left(\delta_{D}\right) \\
A_{y} \cos \left(\phi_{A}+\delta_{A}\right)+B_{y} \cos \left(\phi_{B}+\delta_{B}\right)+C_{y} \cos \left(\phi_{C}+\delta_{C}\right)+D_{y} \cos \left(\phi_{D}+\delta_{D}\right)
\end{array}\right) \\
\mathbf{q}=-\left(\begin{array}{c}
A_{x} \sin \left(\delta_{A}\right)+B_{x} \sin \left(\delta_{B}\right)+C_{x} \sin \left(\delta_{C}\right)+D_{x} \sin \left(\delta_{D}\right) \\
A_{y} \sin \left(\phi_{A}+\delta_{A}\right)+B_{y} \sin \left(\phi_{B}+\delta_{B}\right)+C_{y} \sin \left(\phi_{C}+\delta_{C}\right)+D_{y} \sin \left(\phi_{D}+\delta_{D}\right)
\end{array}\right),
\end{gathered},
$$

while the ellipse parameters are still described by equation 4 . 


\section{EXPERIMENTAL RECORDING}

To create the superimposition of four beams, two Savart plates ${ }^{13,14}$ were used.

The utility of the Savart plate is to divide one incident circularly polarized beam into two linearly orthogonally polarized beams with the same intensity. Assuming an incident beam uniformly polarized under normal incidence, the emerging beams are also at normal incidence with respect to the Savart plate and there is no phase difference between them.

The recording setup contains the following elements (Figure 6):

- a UV laser at $325 \mathrm{~nm}$

- a beam expander

- a linear UV polarizer with a vertical orientation $P_{1}$

- two quarter-wave plates

- two Savart plates.

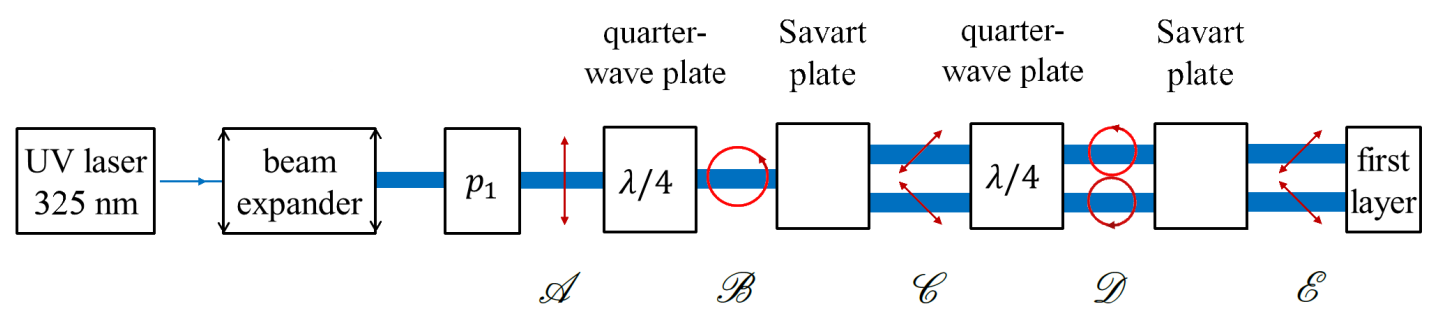

Figure 6. Representation of the recording setup. The beams are pictured in blue and their polarization states are pictured by red arrows, experimentally the beams are larger and a superimposition region exist.

The first quarter-wave plate converts the incident beam (Figure $7 \mathscr{A}$ ) to a circularly polarized one (Figure 7 $\mathscr{B})$. The first Savart plate divides the incident beam into two linearly polarized beams with orthogonal linear polarizations (Figure $7 \mathscr{C}$ ), the beams being separated following the vertical. The second quarter-wave plate converts the incident beam into circularly polarized beams of opposite handedness (Figure $7 \mathscr{D}$ ). Finally, the second Savart plate divides the two incident beams into four beams linearly polarized (Figure $7 \mathscr{E}$ ). The separation will follow the horizontal and the beams are in a square configuration. 


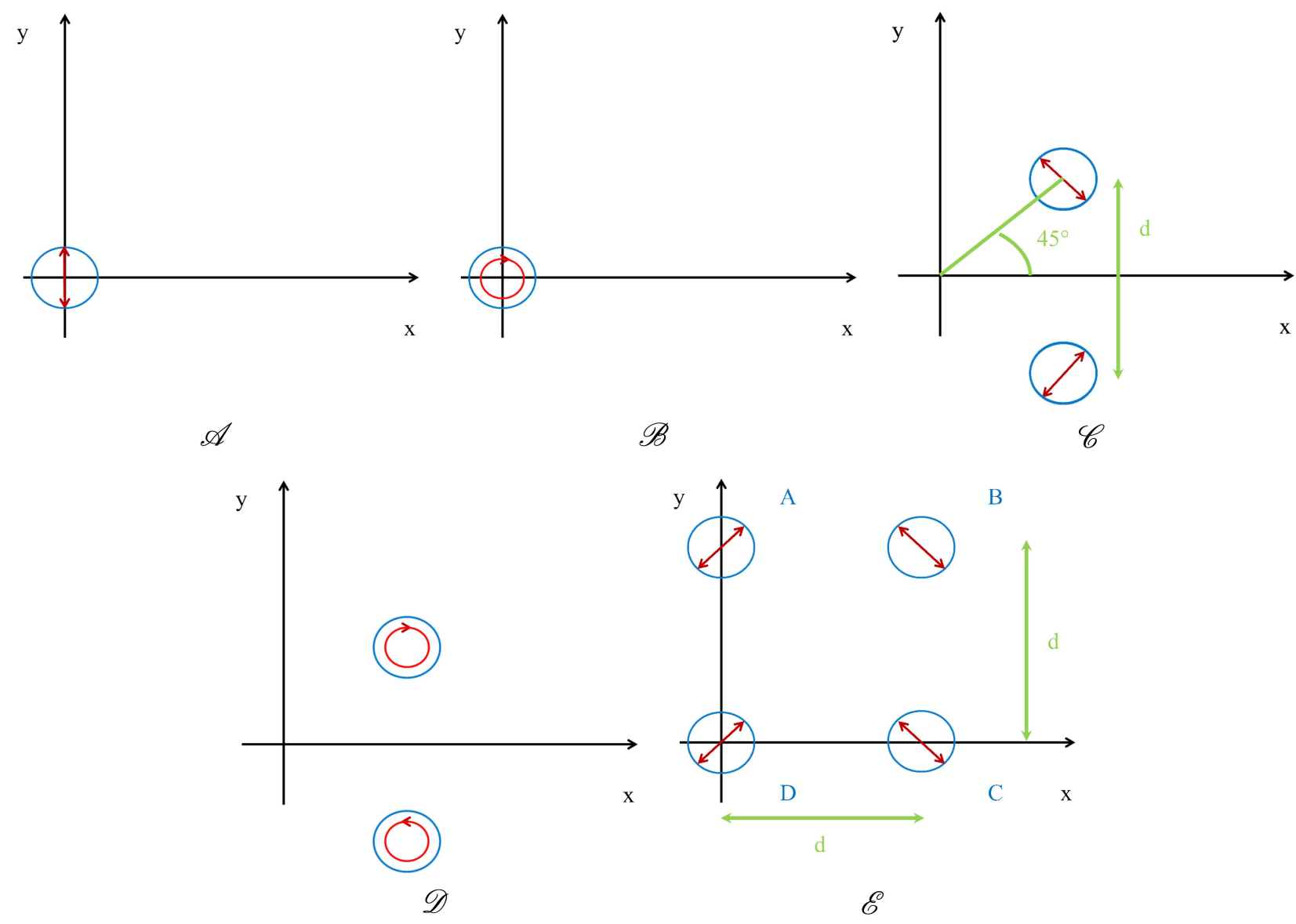

Figure 7. Representation of the positions of the beams and their polarization states through the polarization part of the recording setup. The beams are pictured by blue circles and their polarization states by red arrows. The distance between the beams after the Savart plate is $d$. Experimentally, the beams are larger and a superimposition area of the four beams exists.

Before recording the prototypes, the Stokes parameters of the recording field were measured to determine the characteristics of the local polarization ellipse.

The stokes parameters and the ellipse characteristics are linked by: $:^{9,15}$

$$
\begin{aligned}
& S_{0}=I_{1}+I_{2} \\
& S_{1}=\left(I_{1}-I_{2}\right) \cos (2 \alpha) \\
& S_{2}=\left(I_{1}-I_{2}\right) \sin (2 \alpha) .
\end{aligned}
$$

Where $I_{1}$ is the intensity following the largest semi axis of the polarization ellipse, $I_{2}$ the intenisty following the smallest one and $\alpha$ is the angle formed by the largest axis and the horizontal.

We computed the orientation of the ellipse largest axis $\alpha$, the normalized intensity $S_{0}^{\prime}=\frac{S_{0}}{\max \left(S_{0}\right)}$ and the directionality parameter $d_{p}=1-\frac{I_{2}}{I_{1}}$. The parameters are presented in Figure 8 . 


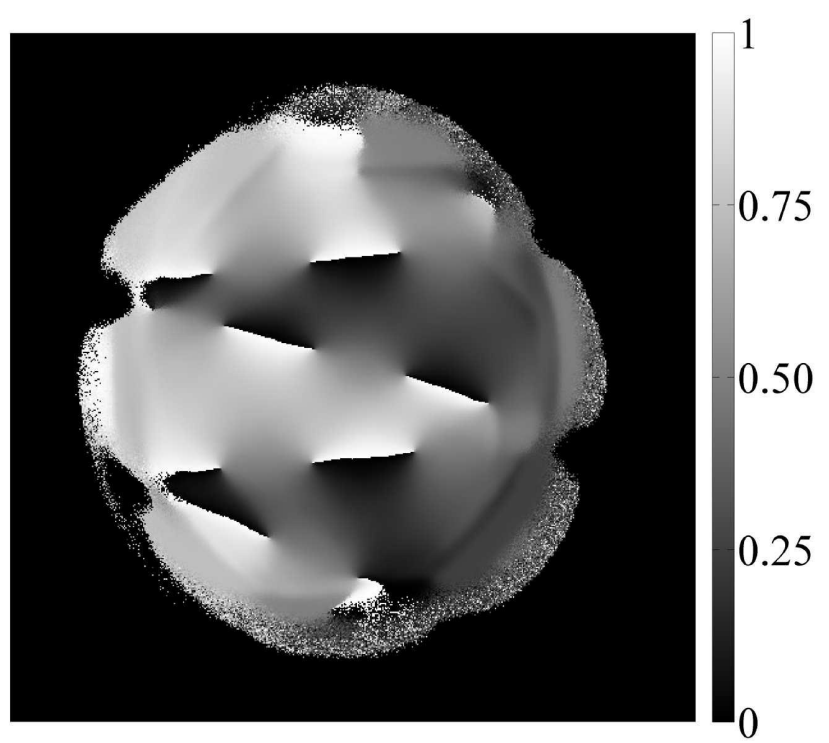

(a)

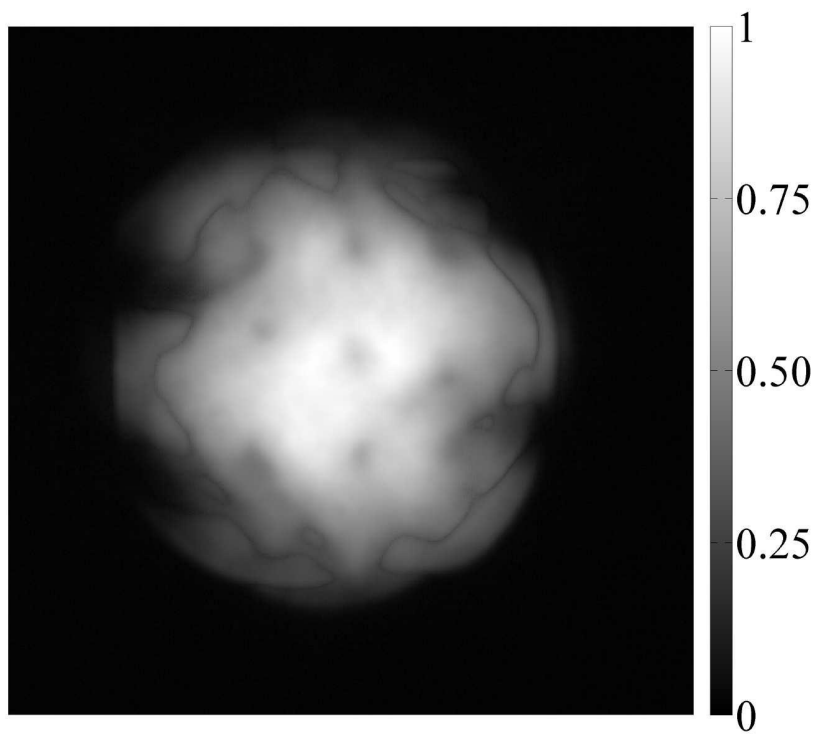

(b)

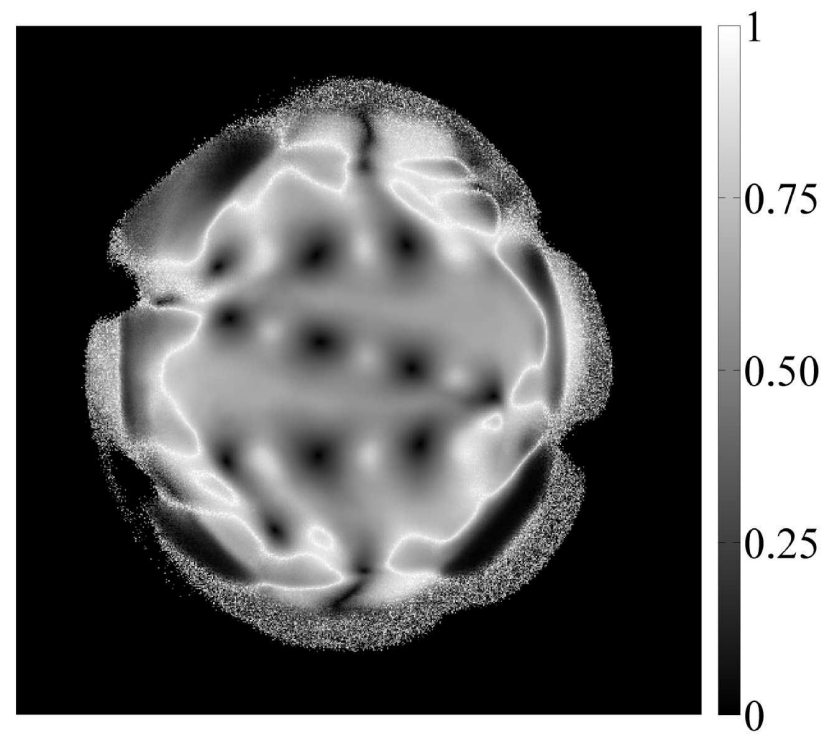

(c)

Figure 8. Representation of the recording field parameters. (a) Pictures the orientation of the electric field $\alpha$, (b) represents the normalized intensity of the recording field $S_{0}^{\prime}$ and (c) details the directionality $d_{p}$.

Thanks to the measurement of $\alpha$ we can observe that:

- several rotation centers are present inside the recording field,

- the rotation centers are aligned on several lines,

- the distance between two rotation centers is about $1.5 \mathrm{~mm}$,

- around a rotation center, the electric field rotates by $180^{\circ}$. It is the characteristics of charge one vortex retarders. 
Using the orientation of the electric field, a half-wave plate was simulated. The orientation of its fast axis corresponds to the orientation of the electric field. The transmitted intensity of the half-wave plate between two crossed polarizers is presented in Figure 9.

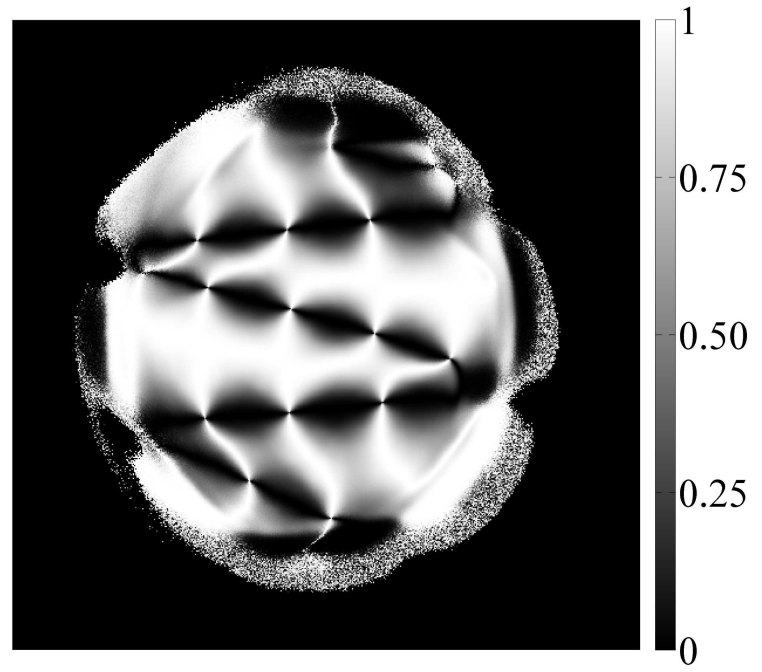

Figure 9. Transmitted intensity of the expected half-wave plate between two linear polarizers.

The other characteristics: $S_{0}^{\prime}$ and $d_{p}$ decrease near the rotation centers. Due to these decreases, the polymers of the first layer may not be properly aligned during the exposure. To determine the recording limits, several retarders were recorded with elliptically polarized beams with the setup described in Figure 10.

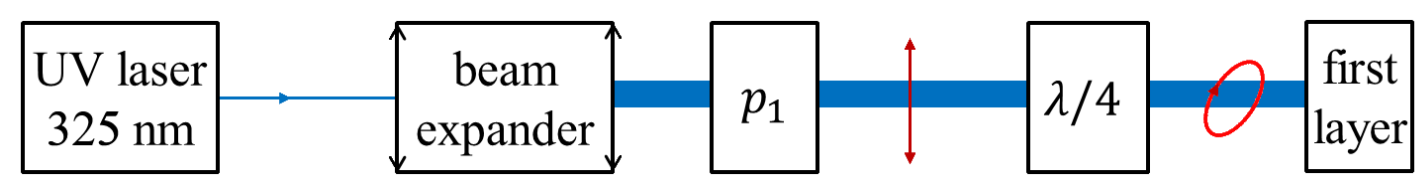

Figure 10. Representation of the recording setup, the beam is pictured in blue and the polarization is represented by red arrows.

From one retarder to the other, the quarter-wave plate was rotated along its normal to change the recording polarization. With this system, we found that the limit depends on the quality of the alignment of the setup. For the current setup, it corresponds to the polarization achieved when the axis of quarter-wave plate forms an angle of $43^{\circ}$ with the direction of the incident polarization. Thanks to this measurements, we were able to compute the size of the possible misorientation areas near the rotation centers, to include them in the computation of the transmitted intensity. Figure 11 presents the transmitted intensity of the expected half-wave plate between two crossed polarizers where the areas of misorientation are pictured by grey disks.

It can be observed that the radius of the largest radius of the misorientation areas is about $10 \%$ of the distance between two rotation centers. 


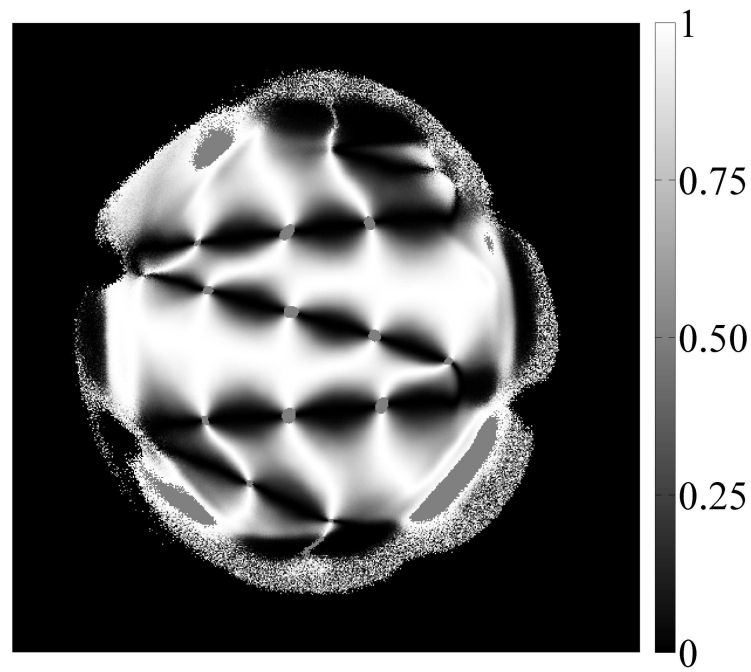

Figure 11. Numerical simulation of the transmitted intensity with area of misorientation. The polarization of the beam after the disorientation areas was computed as an unpolarized beam. Therefore, the transmitted intensity is equal to half of the incident one and grey disks appear on the picture.

\section{FIRST PROTOTYPES}

Finally, two prototypes were recorded using this system. The thickness of the birefringent layer was chosen to match the half-wave condition near $600 \mathrm{~nm}$. Figure 12 presents several pictures of the transmitted intensity of the prototypes between two linear polarizers with a white source.

As expected, the transmitted intensity exhibits the distinctive shape of a retarder containing several charge one vortices. Around a rotation center, there are two bright and two dark areas. The distance between two rotation centers is approximately $1.5 \mathrm{~mm}$. A first estimation of the radius of the misorientation area was performed. It showed that the radius is also approximately about $10 \%$ of the distance between two rotation centers $(150 \mu \mathrm{m})$. Moreover, the pattern of the transmitted intensity for the experimental case is really similar to the one obtained thanks to the measurement of the recording electric field.

\section{CONCLUSIONS AND PERSPECTIVES}

In this paper we presented the first prototypes of vortex retarders exclusively recorded by polarization holography. The principle of polarization holography was presented as well as its application with four beams.

The recording setup was exposed, the measurements of the recording field and the simulations of the expected retarder were detailed. Finally the prototypes were shortly analyzed, their characteristics correspond to the ones expected by the measurements of the recording field.

In the future, a deeper analysis of the first prototypes will be performed to achieve a better accuracy on the distance between two rotation centers and on the radius of the misorientation areas.

Improvements on the recording setup will also be performed. They will allow to easily modify the distance between two rotation centers and to reduce the radius of the misorientation areas compared to the distance between two rotation centers. Finally, a new generation of retarders will be recorded with this setup and they will be measured. 

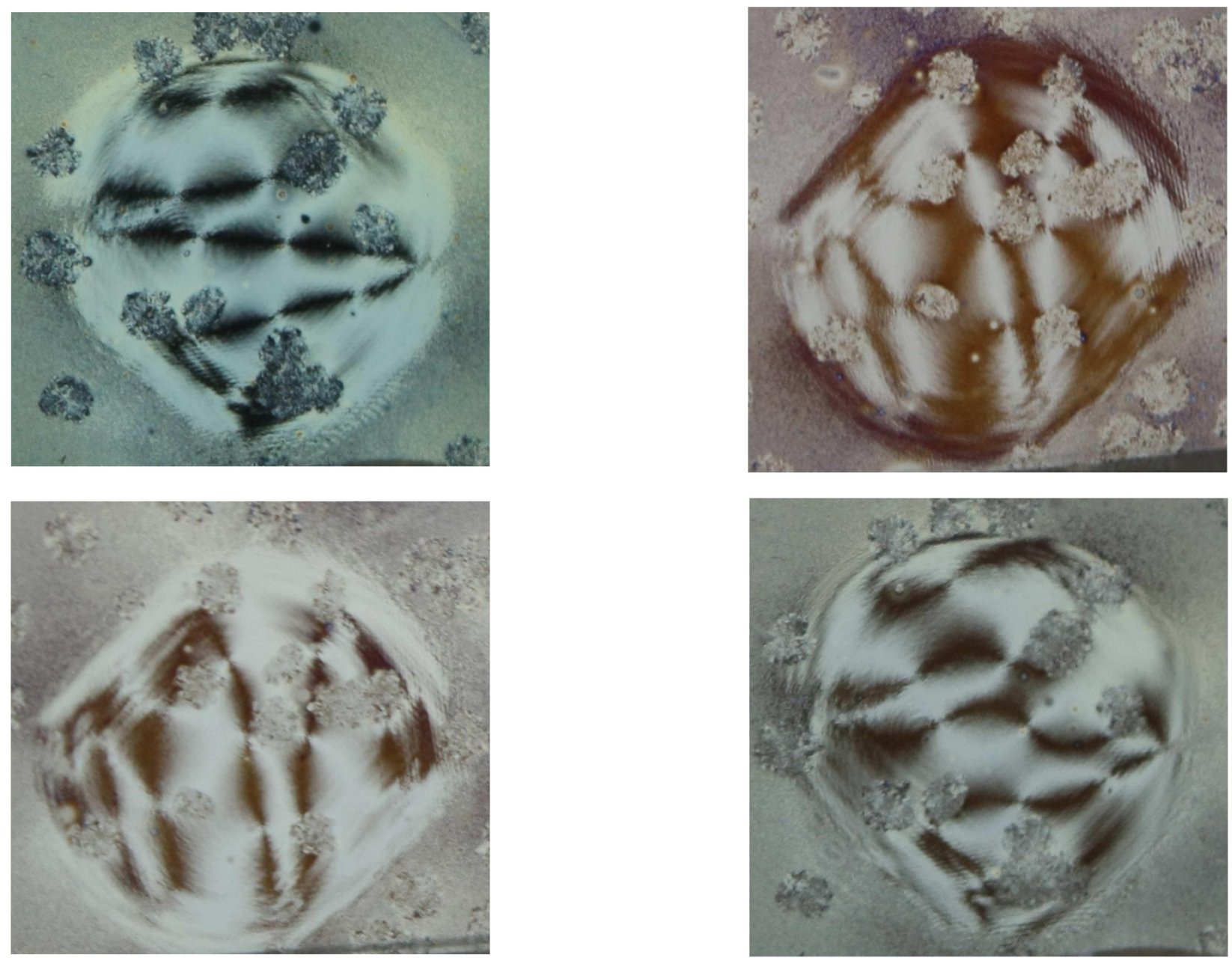

Figure 12. Pictures of the prototypes between two linear polarizers and a white source. The pictures were taken for different orientation between the polarizers. The imperfections are due to dust on the LCP layer or oxidation of this layer during the second spin-coating process.

\section{ACKNOWLEDGMENTS}

The author was funded by the "Fond pour la formation à la Recherche dans l'Industrie et l'Agriculture" (FRIA). The author would like to thanks Loïc Lichawski for his help during his work.

\section{REFERENCES}

[1] Niv, A., Biener, G., Kleiner, V., and Hasman, E., "Manipulation of the pancharatnam phase in vectorial vortices," Opt. Express 14(10), 4208-4220 (2006).

[2] Mawet, D., Serabyn, E., Liewer, K., Burruss, R., Hickey, J., and Shemo, D., "The vector vortex coronagraph : laboratory results and first light at palomar observatory," Astrophys. J. 709(1), 53-57 (2010).

[3] Mawet, D., Riaud, P., Absil, O., and Surdej, J., "Annular groove phase mask coronagraph," Astrophys. J. 633(2), 1191-1200 (2005).

[4] Mawet, D., Riaud, P., Surdej, J., and Baudrand, J., "Subwavelength surface-relief gratings for stellar coronagraphy," Appl. Opt. 44(34), 7313-7321 (2005).

[5] Lerman, G. M. and Levy, U., "Effect of radial polarization and apodization on spot size under tight focusing conditions," Opt. Express 16(7), 4567-4581 (2008). 
[6] Zhan, Q., "Cylindrical vector beams: from mathematical concepts to applications," Adv. Opt. Photon. 1(1), $1-57$ (2009).

[7] Zhan, Q., "Trapping metallic rayleigh particles with radial polarization," Opt. Express 12, 3377-3382 (2004).

[8] Piron, P., Blain, P., Habraken, S., and Mawet, D., "Polarization holography for vortex retarders recording," Appl. Opt. 52(28), 7040-7048 (2013).

[9] Kilosanidze, B. and Kakauridze, G., "Polarization-holographic gratings for analysis of light. 1. analysis of completely polarized light," Appl. Opt. 46(7), 1040-1049 (2007).

[10] Hecht, E., [Optique], Pearson Education France, Paris, fourth ed. (2005).

[11] Piron, P., Blain, P., and Habraken, S., "Polarization measurement with space-variant retarders in liquid crystal polymers," in [Polarization Science and Remote Sensing V], Proc. SPIE 8160, 81600Q-81600Q7 (2011).

[12] Blain, P., Piron, P., Renotte, Y., and Habraken, S., "An in-line shearography setup based on circular polarization gratings," Optics and Lasers in Engineering 51, 1053-1059 (2013).

[13] Françon, M. and Mallick, S., [Polarization interferometers: applications in microscopy and macroscopy], Wiley-Interscience London, New York (1971).

[14] Blain, P., Michel, F., Y.Renotte, and Habraken, S., "Using a savart plate in optical metrology," in [Interferometry XV: Applications], Proc. SPIE 7791, 779107-779107-8 (2010).

[15] Schaefer, B., Collet, E., Smyth, R., Barret, D., and Fraher, B., "Measuring the stokes polarization parameters," Am. J. Phys. 75, 163-168 (2007). 